\title{
Deciphering sarcomatoid RCC
}

Findings of a new study that analysed the genomic and molecular characteristics of sarcomatoid renal cell carcinoma (sRCC) show that this disease is not a homogeneous RCC subtype but can be subdivided according to underlying parental RCC subtypes. These data could help in treatment selection and outcome prediction, as well as in research into new therapeutic strategies.

"RCC with sarcomatoid or rhabdoid morphologies, which can be detected by pathologists under microscopic examination, constitute the most aggressive and therapy-resistant group of RCC," explains senior author Kanishka Sircar from the MD Anderson Cancer Center in Texas, USA. "Following two previous papers, we now used multiplatform molecular profiling to interrogate the most salient genomic aberrations in sRCC using samples from our own centre and data from The Cancer Genome Atlas."

The team found that the mutational load of sRCC and RCC was similar, as was the mutational load in cancer-related genes between epithelioid and spindle cell histologies. Notably, transcriptional data showed that sRCC segregated according to the parent RCC subtype (clear cell RCC, chromophobe RCC, and papillary RCC) rather than to epithelioid or spindle cell components. Upregulation of TGF $\beta$ signalling was seen in all sRCC subtypes. Compared with clear cell RCC, sRCC of that subtype had fewer deletions at 3p21-p25 (encoding VHL, PBRM1, SETD2, and BAP1), a lower rate of concurrent mutation and copy loss for $\mathrm{VHL}$ and PBRM1, and more mutations in PTEN, TP53, and RELN; mutations in these three genes were predictive of sarcomatoid clear cell RCC and poor prognosis.

"RCC comprises many more molecular entities than described in the current WHO classification and appreciable by microscopic examination," concludes Sircar. "We plan to develop a molecular signature of sRCC for diagnostic applications and to study targets and activated pathways in sRCC that can be exploited for therapeutic benefit."

Clemens Thoma

ORIGINAL ARTICLE Wang, Z. et al. Sarcomatoid renal cell carcinoma has a distinct molecular pathogenesis, driver mutation profile and transcriptional landscape. Clin. Cancer Res. http://dx.doi.org/10.1158/1078-0432.CCR-17-1057 (2017) 